\title{
SPECIAL ARTICLE SPR Perspectives: scientific opportunities in the Environmental influences on Child Health Outcomes Program
}

Megan E. Romano ${ }^{1}$, Jessie P. Buckley ${ }^{2,3}$, Amy J. Elliott ${ }^{4}$, Christine C. Johnson ${ }^{5}$, Nigel Paneth ${ }^{6,7}$ and on behalf of program collaborators for Environmental influences on Child Health Outcomes

Drawing upon extant data from existing pediatric cohorts and new follow-up of a diverse set of pediatric cohorts from across the United States, the Environmental influences on Child Health Outcomes (ECHO) Program creates the opportunity for novel and innovative investigations of many previously inaccessible scientific questions in the area of child health. We describe how the large sample size, diversity of participants, emphasis on team science, and infrastructure for improving research methodology make the ECHO Program a major research resource for improving our understanding of early life determinants of childhood health and wellbeing. Pediatric researchers leverage the unique features of the ECHO Program to address research questions with the potential to yield far-reaching and long-term impacts on child health.

Pediatric Research (2022) 92:1255-1261; https://doi.org/10.1038/s41390-021-01577-5

\section{IMPACT:}

- The ECHO Program unites pediatric cohorts from across the United States, allowing for investigations of compelling research questions that were previously infeasible due to limited sample sizes or lack of participant diversity.

- The focus of the ECHO Program on team science, solution-oriented research, and methodological innovation propels novel scientific investigations that are responsive to the needs of a wide range of stakeholders.

- Features of the ECHO program's infrastructure poise its investigators to rapidly launch research endeavors that are responsive to time-sensitive and critical needs within the realm of pediatric research.

\section{INTRODUCTION}

Despite major advances in pediatric research in the twentieth and twenty-first centuries, many unanswered questions remain about how the prenatal and early life environments influence children's health and well-being. The Society for Pediatric Research recently published a perspectives paper promoting the need for research into the interrelations of social and environmental determinants of health that may be translated into interventions targeting the social needs of children, ${ }^{1}$ and both the American Academy of Pediatrics and the American College of Obstetricians and Gynecologists have formally recognized the influence of exposure to environmental factors in early life on both the short- and long-term health of children. ${ }^{2,3}$ Defined broadly, the environment includes the totality of early life surroundings, encompassing home, neighborhood, socioeconomic status, diet, and psychosocial factors, as well as more traditional environmental exposures such as air pollution, toxic metals, and chemical toxicants. Large prospective pregnancy and birth cohorts are needed to inform early interventions by elucidating both risk factors for poor health and health-protective factors promoting resilience across the life course. However, large-scale prospective cohort studies pose logistical, financial, and methodological challenges for individual researchers, necessitating a synergistic effort across scientific disciplines to create research consortia that can address scientific questions that were previously infeasible due to constraints of sample size, expertise, or infrastructure. The Environmental influences on Child Health Outcomes (ECHO) Program holds much promise for investigating many of these previously inaccessible questions, drawing upon already collected information from a diverse set of established pediatric cohorts and providing a platform to prospectively follow children through an integrated data collection approach., ${ }^{4,5}$ Given its diversity, focus on team science and "Big Wins," and provision of a platform to improve pediatric research methodology, ECHO's potential as a research resource for improving our understanding of how early life environmental factors contribute to childhood health and wellbeing is currently unparalleled in the United States. This article (part of a series of companion papers detailing aspects of the

\footnotetext{
${ }^{1}$ Department of Epidemiology, Geisel School of Medicine, Dartmouth College, Hanover, NH, USA; ${ }^{2}$ Department of Environmental Health and Engineering, Bloomberg School of Public Health, Johns Hopkins University, Baltimore, MD, USA; ${ }^{3}$ Department of Epidemiology, Bloomberg School of Public Health, Johns Hopkins University, Baltimore, MD, USA; ${ }^{4}$ Avera Research Institute Center for Pediatric and Community Research, Sioux Falls, SD, USA; ${ }^{5}$ Department of Public Health Sciences, Henry Ford Health System, Detroit, MI, USA; ${ }^{6}$ Department of Epidemiology and Biostatistics, College of Human Medicine, Michigan State University, East Lansing, MI, USA and ${ }^{7}$ Department of Pediatrics and Human Development, College of Human Medicine, Michigan State University, East Lansing, MI, USA

Correspondence: Megan E. Romano (megan.e.romano@dartmouth.edu)

See Acknowledgments for a full listing of collaborators.
}

Received: 28 January 2021 Accepted: 11 February 2021

Published online: 25 May 2021 


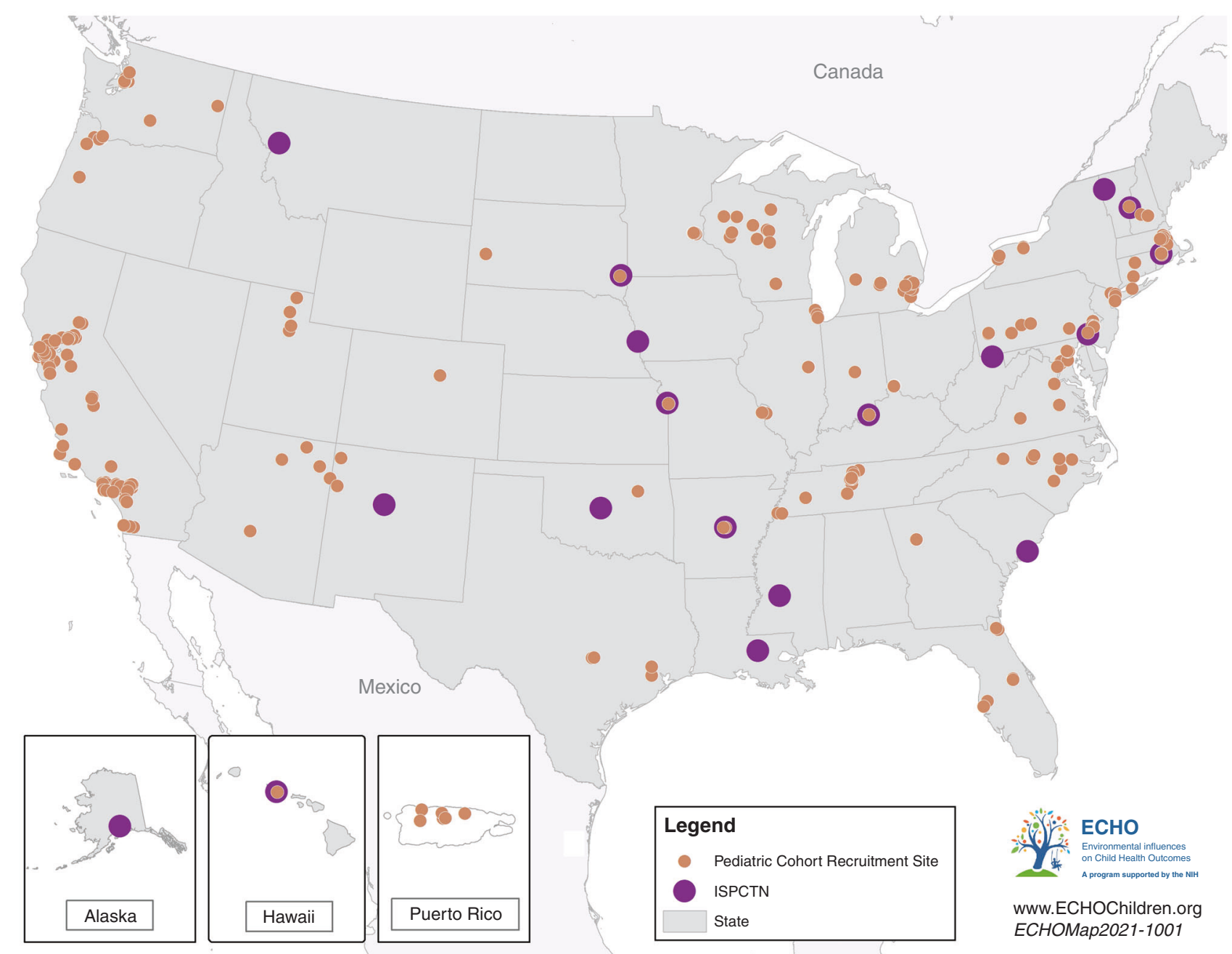

Fig. 1 Map of ECHO Pediatric Cohort Recruitment Sites and the Institutional Development Award (IDeA) States Pediatric Clinical Trials Network (ISPCTN). Map of ECHO Pediatric Cohort Recruitment Sites and the Institutional Development Award (IDeA) States Pediatric Clinical Trials Network (ISPCTN).

ECHO Program) focuses on the scientific opportunities within ECHO.

\section{THE ECHO PROGRAM}

As detailed in the accompanying article by LeWinn et al., ${ }^{6}$ the ECHO Program was designed to produce a national resource, available to scientific investigators both within and outside the network, to leverage the wealth of information collected during the prenatal and early life stages by many sets of investigators, as well as by conducting longer term follow-up of established cohorts. The ECHO Program's 69 pediatric cohorts include $>57,000$ children and families, representing most geographic regions across the United States (Fig. 1).

The ECHO-wide Cohort Data Collection Protocol includes new data and biological samples collected by each cohort and is designed to prospectively investigate the influence of early life exposures on child health and development with a focus on five areas of health: pre-, peri-, and early postnatal; obesity; neurodevelopment; breathing; and positive health. ${ }^{7}$ ECHO has engaged key stakeholder groups to help design the protocol and inform analytic proposals with the end user in mind. The scientific community will soon have access to the wealth of data already collected during the prenatal period and early life stages. The extant cohort data have already been mapped to each data element of the ECHO-wide protocol, and the challenging but achievable process of data harmonization across different assessment instruments and analyses will merge the past with the future (see the accompanying article by Jacobson et al. ${ }^{8}$ ).

Compiling and harmonizing extant and new data from this large and diverse population of children and their families allows for a relatively rapid start-up of large-scale studies on the influences of the environment on child health and development. These cohorts have a degree of diversity rarely found even in large prospective studies. Included are both inner city and rural populations that have traditionally both been medically underserved and economically deprived. Because the bulk of pediatric research is based on convenience samples available to academic investigators, many of these populations have not traditionally been well represented in pediatric research. ECHO includes cohorts that have been established among Latino and Native American Populations, and some $14 \%$ of children in ECHO cohorts are Black/African American and 24\% are Hispanic/Latino.

Inasmuch as rural children have generally been underrepresented in clinical trials, the ECHO Program has joined with the Institutional Development Award (IDeA) States program to form the integrated ECHO IDeA States Pediatric Clinical Trials Network (ECHO ISPCTN). This network includes 17 states and invigorates clinical trial expertise in these locations to form new pediatric research centers in partnership with academic 
Table 1. Solution-oriented potential "Big Wins" identified by the ECHO Program.

Title

Epidemiology of incident asthma

Assessing exposures to novel chemicals among pregnant women in ECHO: a pilot to inform studies of associations with child health outcomes

Smoking and autism disorder

Inadequate and excessive intake of micronutrients in pregnancy

Early childhood asthma and obesity incidence

A participant-level analysis of the long-term development outcomes of prenatal opioid exposure

Children's sleep and life satisfaction

Geographical patterns of growth trajectories in children

Prenatal opioid use and psychosocial adversity associated with behavioral

trajectories from 2 to 6 years

The effect of social policies on the health of children in vulnerable populations
Project lead, institution

Christine Johnson, Henry Ford Health System

essie Buckley, Johns Hopkins University; Tracey Woodruff, University of California, San Francisco

Irva Hertz-Picciotto, University of California, Davis

Katherine Sauder, University of Colorado, Denver

Leda Chatzi, University of Southern California

Elisabeth Conradt, University of Utah

Courtney Blackwell, Northwestern University

Dana Dabelea, University of Colorado, Denver

Julie Hofheimer, University of North Carolina at Chapel Hill

Rita Hamad, University of California, San Francisco; Kaja LeWinn, University of California, San Francisco institutions (Fig. 1). The goals of the ECHO ISPCTN are to increase access of medically underserved and rural pediatric populations to clinical trials and to build capacity to conduct pediatric clinical trials through the creation of infrastructure and provision of professional development for faculty and support teams. ${ }^{9}$ In conjunction with data collected from ECHO Pediatric Cohorts, the creation of a pediatric clinical trials infrastructure provides the opportunity for researchers to answer large, complex questions about child health and more readily inform health policy in the United States. Further, these synergistic research efforts allow for the integration of findings from observational research into the implementation of interventional trials.

Team science approach

Increasingly complex research questions necessitate collaborations across disciplinary, organizational, and geographic boundaries. Likewise, advances in technology, including omics (e.g., genomics, proteomics) and high-dimensional statistical analyses, necessitate the integration of a broader range of scientific expertise into individual studies. ${ }^{10}$ Several ECHO components promote the implementation of team science and are critical to the program's success. The Data Analysis Center (DAC), located at Johns Hopkins University and RTI International, provides guidance in the design and conduct of ECHO studies, manages the integration and quality of data across the pediatric cohorts, and assists in the statistical analysis. ${ }^{11}$ Likewise, ECHO leverages the expertise of its many investigators by establishing working groups to help achieve its operational and research goals. ECHO working groups are focused on facilitating collaboration, aligning scientific objectives, and accelerating decision-making. ${ }^{6}$ The working groups are supplemented by focused task forces engaged in the creation of specific protocols to support the advancement of $\mathrm{ECHO}$ science. For example, the Wearables Task Force creates protocols for the integrations of wearable technology to assess cardiovascular metrics, physical activity, and sleep into ECHO. Additional task forces focus on the microbiome, return of results to participants, and COVID-19, among other topics. These working groups and task forces incorporate scientists from many disciplines and provide the ECHO Program with a strong foundation to support intellectual discourse and underpin the values of team science that run throughout all aspects of the program. $^{12}$

\section{ECHO Big Wins}

A key feature of ECHO's Strategic Plan is to foster solution-oriented potential "Big Wins," opportunities to leverage the unique aspects of the ECHO Program to create timely responses to specific research questions that will influence long-term child health and disease risk. The task force established a process by which to select 1-3 potential Big Wins per year from the many Analysis Proposals approved by the ECHO Steering Committee. These research questions lie at the intersection of three key elements: investigator passion, feasibility, and end-user stakeholder priorities. Investigator passion refers to channeling investigator expertise and creativity to conceptualize, initialize, and develop compelling and high-impact studies. Feasibility is a necessary component for research proposals that can be executed using ECHO's data, biological samples, and data analytic resources. Finally, Big Wins are designed to answer research questions identified by end-user stakeholders (including parents, clinicians, and policy makers for child health) as priorities to fill important gaps that will influence programs, policies, and practices to improve child health. Big Wins are expected to have rigorous analytic plans, and to focus on solutions with a sizeable population attributable risk (i.e., a large impact on a large population). Once selected, potential Big Wins are allocated additional $\mathrm{ECHO}$ resources to ensure timely completion, including extra support from the Coordinating Center, Patient Reported Outcomes Core, and DAC. To date, ECHO has recognized ten potential Big Wins that focus on a wide range of topics covering ECHO's key outcome focus areas (Table 1), including recently published research on pediatric sleep quality. ${ }^{13}$ Highlights are presented below from two of the selected projects that demonstrate the breadth and depth of solution-oriented research opportunities in $\mathrm{ECHO}$.

Big Win example 1

The first Big Win example is an investigation of the epidemiology of incident asthma in pediatric populations in the United States. This work illustrates the guiding principles of solution-oriented research in $\mathrm{ECHO}$, incorporating data from $32 \mathrm{ECHO}$ cohorts and providing much needed descriptive epidemiology related to the incidence rates of asthma among children across the country. The work will inform future prevention strategies geared at improving the respiratory health of children from a diverse set of backgrounds.

Investigator passion. The initial idea for the Big Win was generated during a breakout session for investigators interested in Airways Outcomes at the very first Steering Committee meeting in November 2016. ECHO investigators were assigned to different tables to brainstorm potential ideas for eventual ECHO studies. The launch of this Big Win was energized by the idea that the large numbers of children included in $\mathrm{ECHO}$ and the inclusion of 
respiratory diseases as one of the primary outcomes would allow for the retrospective construction of a "surveillance system" for asthma across many diverse populations. While asthma is the most common chronic disease among children, it is not a reportable disease. Thus, basic descriptive epidemiology has been lacking. In particular, incidence rates have been difficult to estimate, and are of course the most useful epidemiological measure for stimulating etiological hypotheses to suggest which populations have the most critical need for preventative interventions.

Feasibility. At the inception of this project, the DAC had acquired detailed information about the specific data available across the various $\mathrm{ECHO}$ pediatric cohorts. In addition to cohorts specifically designed to study asthma, many of the pediatric cohorts had inquired about asthma diagnoses during follow-up as part of a general health screen. At the time, data were beginning to be compiled and harmonized centrally, but data use agreements and other infrastructure elements were not yet completed. Thus, the decision was made to perform a distributed collective analysis whereby code was developed by the DAC and sent to the cohorts to be run at their home institution rather than pooling data centrally for analysis. Results from individual cohorts were then sent back to the DAC and combined using a meta-analysis approach. A total of 32 cohorts had enrolled 24,635 children born at $\geq 34$ weeks gestation and followed until at least their fifth birthday with data collected on physician diagnosis of asthma.

End-user stakeholder priorities. This study provides data for the recently renewed focus on preventing the development of asthma. Early development of this condition has been shown to result in suboptimal lung function over the entire life course. ${ }^{14,15}$ Despite the development of new medications and environmental and behavioral approaches to improve asthma control, many of the drugs are exceedingly expensive, and an asthma diagnosis has a negative impact on daily life. ${ }^{16} \mathrm{~A}$ wide range of stakeholdersincluding clinicians, parents, teachers, and payers-have turned their attention to the elusive goal of asthma primary prevention and the development of effective interventions. The data provided in this incidence study demonstrated that children who have a parent with asthma had incidence rates 2-3 times higher in the first 4 years of life. In this same age group, incidence rates were remarkably higher in African-American children. Rates in girls were rather steady over childhood, whereas boys had higher rates in the preschool years, which then declined. These results suggest that etiological factors are present very early in life and have differential effects in population subgroups. The findings also indicate that it is a priority to develop tailored interventions for the group with the highest rates: preschool African-American children with a family history of asthma. ${ }^{17}$

\section{Big Win example 2}

Another potential Big Win for ECHO is a project to measure exposure to priority emerging chemicals in a large sample of pregnant women across the United States and evaluate the chemicals' effects on child health. Although pregnant women are potentially exposed to thousands of chemicals through food, water, air, dust, and personal care products, ${ }^{18}$ nationally representative data on chemical exposures are available from the National Health and Nutrition Examination Survey (NHANES) for only $\sim 350$ of the $>40,000$ chemicals actively approved for use in the United States. ${ }^{19}$ This project was selected as a potential Big Win given that it fills an important data gap and supports solutionoriented research in $\mathrm{ECHO}$.

Investigator passion. Through new collaborations sparked by ECHO's Chemical Exposures Working Group, ECHO investigators sought to identify and prioritize chemical exposures of high relevance to $\mathrm{ECHO}^{\prime}$ s health outcome focus areas. In addition to characterizing chemicals measured in NHANES, ${ }^{20}$ the investigators conducted an extensive review to identify novel chemicals and prioritize them based on the likelihood of exposure, the potential for adverse health effects, and biomarker availability. ${ }^{21}$ Through this process, the investigators recommended 36 chemicals as a high priority for biomonitoring in ECHO. These findings informed the design of the Big Win pilot study, leveraging the investigators' passion to address key gaps in research into in utero exposures to environmental chemicals. The first step of this study will measure urinary biomarkers of approximately 100 compounds among 180 pregnant women from nine geographically diverse $\mathrm{ECHO}$ cohorts.

Feasibility. This project is feasible due to the availability of data and samples from ECHO cohorts as well as the ECHO Program's Human Health Exposure Analysis Resource (HHEAR; formerly the Child Health Exposure Analysis Resource). ${ }^{22}$ For this Big Win project, HHEAR's Targeted Analysis Laboratory developed efficient, high-throughput methods for multiple chemical extraction and measurement to quantify a large number of chemicals, including both novel and legacy compounds (alternative flame retardants, alternative plasticizers, aromatic amines, environmental phenols, and pesticides).

End-user stakeholder priorities. For most of the specified chemicals, this study will be the first to measure exposures among pregnant women in the United States. Measured novel chemical exposures during pregnancy will provide important data about exposures from common products and sources that have implications for child health. This may lead to individual-level behavior change, clinician-level guidance, and population-level interventions. The long-term goal of this project is to fill knowledge gaps regarding exposures and pediatric health effects to inform science and expedite programs, policies, and practices for protecting children's health.

\section{DEVELOPMENT OF TOOLS AND METHODS FOR PEDIATRIC RESEARCH}

ECHO also supports the development of pediatric research tools and technologies with utility beyond the program cohorts. Initiated in 2016, the Opportunities and Infrastructure Fund (OIF) is a grant mechanism funded through the National Institutes of Health and administered through the ECHO Coordinating Center. Early-stage investigators from the pediatric cohorts and other ECHO components are eligible to apply for these pilot funds, which allow for the validation and development of novel tools, technologies, and methods with broader applications to pediatric research beyond ECHO studies. OIF projects further emphasize transdisciplinary research, team science, and collaboration across multiple pediatric cohorts. Three thematic award cycles have been initiated through the OIF mechanism to date. The themes of the research have been (1) validation of measures and integration of technology-based measures; (2) innovations in the collection, analysis, and utilization of biomarkers and novel approaches for the collection and analysis of complex data; and (3) methods to analyze trajectories (e.g., growth, neurodevelopment) or facilitate cross-cohort comparisons and handling missing data (Table 2). Projects from the first cycle of the OIF program are nearing completion, and awardees for the third cycle were announced during the summer of 2020 . Across the cycles, innovative projects seek to improve exposure assessment tools (e.g., use of silicone wristbands as passive exposure monitors), ${ }^{23}$ reduce participant burden (e.g., validate abbreviated forms of longer instruments), ${ }^{24}$ create novel tools for investigating neurodevelopmental outcomes, enhance assessment of air pollution exposures, and develop geospatial indicators of socioeconomic status and greenspace. Further, collection of blood samples in pediatric 
Table 2. Opportunities and Infrastructure Fund projects.

Title

Project principal investigator, institution

Cycle 1: validation of measures and integration of technology-based measures

The remote food photography method: pilot testing a novel technology-based measure of Traci Bekelman, University of Colorado Denver dietary intake in school-age children

The air we breathe: prenatal exposure to air pollutants and child development

Akhgar Ghassabian, New York University

ECHO-wide platform for studying air pollution, temperature, and greenness using satellite remote sensing with daily high-resolution national exposure estimates

Optimizing social communication measurement with the social responsiveness scale

Allan Just, Icahn School of Medicine at Mount Sinai

Attentional mechanisms underlying information processing in a sample of Navajo children

Developing exposure characterization tools to address complex exposures within ECHO

Kristen Lyall, Drexel University

The prenatal poly-substance exposome: validation of multiple chemical exposures on

systemic absorption, and implications for novel serum metabolomics in gestation

Using silicone wristbands as noninvasive, passive environmental monitors to evaluate

seasonal and within-family correlation for environmental exposures

Parallel analysis of placental metabolomics and histology

Sara Nozadi, University of New Mexico

John Pearce, Medical University of South Carolina

Megan Romano, Geisel School of Medicine at

Dartmouth

Raymond Soto, University of Utah

Integration of nonnutritive suck and eye tracking as markers of neurodevelopment across Emily Zimmerman, Northeastern University five ECHO cohorts

Cycle 2: innovations in the collection, analysis, and utilization of biomarkers and novel approaches for the collection and analysis of complex data

Decentralized and reproducible geomarker assessment for multi-site studies

Cole Brokamp, Cincinnati Children's Hospital Medical Center

Seeing the forest for the trees: developing Google street view-based metrics of nature and their influence on health

Novel method for high-frequency molecular biomarker collection

Peter James, Harvard Pilgrim Healthcare Incorporated

Validation of maternal vaginal microbiota signatures in pregnancy: the ECHO Vaginal

Microbiome Consortium

Improving usability of smartphone tools for reporting chemical biomonitoring results

Leveraging transcriptional regulatory networks for exposure analysis

Developing robust untargeted methods for DBS and microsampler analysis in ECHO

Candace Lewis, Translational Genomics Research Institute

Kimberly McKee, University of Michigan

ECHO-relevant hypothesis testing using an accessible and reproducible infrastructure for sharing data

Salivary uric acid, early life stress, and cardiometabolic health

Using oscillometry to identify early life determinants of childhood lung function

Towards improving infant MRI segmentation using convolutional neural network deep-

learning approaches

Amy Padula, University of California, San Francisco Alison Paquette, Institute for Systems Biology Lauren Petrick, Icahn School of Medicine at Mt. Sinai Jerod Rasmussen, University of California, Irvine

Jenna Riis, University of California, Irvine Katharine Hamlington Smith, University of Colorado Denver

Yun Wang, Columbia-Research Foundation for Hygiene

\section{Cycle 3: methods to analyze trajectories or facilitate cross-cohort comparisons and handling missing data}

Cross-cohort mixture analysis: prenatal metals exposure and birth outcomes

Oxidative stress and inflammation biomarkers in relation to birth outcomes in four ECHO Cohorts

Elena Colicino, Icahn School of Medicine at Mt. Sinai

Stephanie Eick, University of California, San Francisco

Per- and poly-fluoroalkyl substances (PFAS) Exposures And Child Health (PEACH) study: using Donghai Liang, Emory University targeted and untargeted exposomics profiling to characterize metabolic pathways and

mechanisms underlying PFAS toxicity on adverse birth and child health outcomes

Identifying dynamic change processes in growth trajectories from infancy to early adolescence

Prenatal secondhand smoke and chemical exposures and offspring markers of obesity in the Andreas Neophytou, Colorado State University ECHO Consortium: transportability of effect estimates across ECHO Cohorts

Improved single and multiple imputation methods (ISAMIM) for missing dichotomous and Fares Qeadan, University of Utah polytomous data

Spatiotemporal characterization of placental growth trajectories: novel measures of fetal programming

The methylome as a predictor of maternal smoking status and childhood lung function trajectory

Ruchit Shah, Placental Analytics, LLC

Lyndsey Shorey-Kendrick, Oregon National Primate Research Center

Leveraging existing newborn screening metabolic data to understand childhood health and Brittney Snyder, Vanderbilt University disease

Medical Center 
populations is challenging, and multiple projects are working to leverage dried blood spots, which require a single heel or finger prick, to drastically reduce the burden of blood collection for young study participants. OIF projects span a wide range of substantive areas within the realm of pediatric research and offer great potential for improving available technologies to address longstanding challenges within pediatric epidemiologic investigations. Collectively, the OIF projects offer much promise to the broader field of pediatric research in support of ECHO's mission "to enhance the health of children for generations to come." 25

Scientific opportunities and potential for rapid response by the ECHO Program

Most birth cohorts are designed to focus intensively in one outcome area. With the usual resource and expertise constraints, it is challenging to collect the necessary in-depth data related to more than one disease. Further, most birth cohorts are powered to address only a few hypotheses in a painstaking fashion, with sample sizes ranging from several hundred to several thousand. Smaller cohorts are usually able to collect more detailed measures more frequently, but have limited statistical power due to sample size. In particular, studying clinical outcomes with relatively low prevalence in the general pediatric population, such as autism spectrum disorder or severe asthma, requires a sample size typically beyond the scope of an individual pediatric cohort. ECHO's design overcomes this implicit limitation in health research. Cohorts within a specific disease outcome area, while individually limited in sample size, have collected comprehensive data concentrating on risk factors and biological measures related to the outcome in question. With the ability to harmonize and pool these rich datasets within an outcome area, ECHO allows a comprehensive analysis of confounding and effect modification and replication using diverse samples no longer constrained by population size, geography, local population characteristics, or institutional investigator expertise.

$\mathrm{ECHO}$ is a nimble data resource capable of addressing the major public health challenges of our time. For example, ECHO is collaborating with the Eunice Kennedy Shriver National Institute of Child Health and Human Development to study neonatal opioid withdrawal syndrome (NOWS). Historically, NOWS has been challenging to study, due to a lack of large prospective cohorts with a robust assessment of potential confounding variables and longitudinal data spanning well beyond infancy. ECHO offers an opportunity to assess long-term neurodevelopmental outcomes among children exposed to opioids in utero, and these observational studies can directly inform clinical trials carried out through ECHO ISPCTN to provide actionable options for intervention and prevention. ${ }^{26}$ More recently, ECHO responded to the COVID-19 pandemic with swift approval of data collection instruments to obtain timely information on health outcomes among those with SARS-CoV-2 and psychosocial and behavioral aspects of life during the pandemic, as well as survey versions tailored to caregivers, pregnant women, and adolescents. ${ }^{27,28}$ In addition, the ECHO ISPCTN implemented a task force to explore options for remote operations, including obtaining informed consent, collecting data, and implementing interventions, particularly those related to COVID-19. ${ }^{27}$ ECHO's nationwide cohort and robust data collection infrastructure have the program uniquely poised to respond to ongoing and novel threats to child health.

\section{CONCLUSIONS}

ECHO represents exciting new opportunities for pediatric research, allowing for the investigation of scientific questions related to less common childhood outcomes and increasing inclusiveness of children participating in research in the United States. By harmonizing extant data and creating a common protocol for continuing follow-up, ECHO has created a powerful data resource for the pediatric research community. Focus on stakeholder priorities allows for the implementation of research that can have a meaningful impact on health policy and inform preventative interventions geared at improving and preserving children's health. Implementation of a team science approach and opportunities for methods development further bolster ECHO's positive impact on the broader pediatric research community. Ultimately, ECHO research serves to promote the health and wellbeing of children, now and in the future.

\section{ACKNOWLEDGEMENTS}

We wish to thank our ECHO colleagues, the medical, nursing, and program staff, as well as the children and families participating in the ECHO cohorts. We also acknowledge the contribution of the following ECHO Program collaborators: Coordinating Center-Duke Clinical Research Institute, Durham, N C: P.B. Smith, K.L. Newby, D.K. Benjamin; Data Analysis Center-Johns Hopkins University Bloomberg School of Public Health, Baltimore, MD: L.P. Jacobson; Research Triangle Institute, Durham, NC: C.B. Parker. We would also like to acknowledge and thank the following: Peter Hoffmann, Beth Harris, and Michelle Schreiner. Research reported in this publication was supported by the Environmental influences on Child Health Outcomes (ECHO) program, Office of The Director, National Institutes of Health, under Award Numbers U2COD023375 (Coordinating Center), U24OD023382 (Data Analysis Center), UH3 OD023275, UH3 OD023382, UH3OD023282, and UH3 OD023285. The content is solely the responsibility of the authors and does not necessarily represent the official views of the National Institutes of Health.

\section{AUTHOR CONTRIBUTIONS}

All authors contributed to the conception and design of this manuscript and participated in its drafting. All authors have provided final approval of the version submitted for publication.

\section{ADDITIONAL INFORMATION}

Competing interests: The authors declare no competing interests.

Consent statement: Patient consent was not required for this review article.

Publisher's note Springer Nature remains neutral with regard to jurisdictional claims in published maps and institutional affiliations.

\section{REFERENCES}

1. Beck, A. F. et al. Perspectives from the Society for Pediatric Research: interventions targeting social needs in pediatric clinical care. Pediatr. Res. 84, 10-21 (2018).

2. Etzel, R. A. \& Balk, S. J. Pediatric Environmental Health (American Academy of Pediatrics, 2019).

3. American College of Obstetricians and Gynecologists. American College of Obstetricians and Gynecologists Committee on Health Care for Underserved Women, American Society for Reproductive Medicine Practice Committee, The University of California, San Francisco Program on Reproductive Health and the Environment. Exposure to Toxic Environmental Agents. Committee Opinion Number 575 https://www.acog.org/clinical/clinical-guidance/committee-opinion/ articles/2013/10/exposure-to-toxic-environmental-agents (2013).

4. Paneth, N. \& Monk, C. The importance of cohort research starting early in life to understanding child health. Curr. Opin. Pediatr. 30, 292-296 (2018).

5. Tylavsky, F. A. et al. Understanding childhood obesity in the US: the NIH Environmental Influences on Child Health Outcomes (ECHO) program. Int. J. Obes. 44, 617-627 (2020).

6. LeWinn, K. Z., Caretta, E., Davis, A., Anderson, A. L. \& Oken, E., Program Collaborators for Environmental influences on Child Health Outcomes. SPR Perspectives: environmental influences on Child Health Outcomes (ECHO) Program: overcoming challenges to generate engaged, multidisciplinary science. Manuscript in preparation (2021).

7. Forrest, C. B., Blackwell, C. K. \& Camargo, C. A. Jr. Advancing the science of children's positive health in the National Institutes of Health Environmental Influences on Child Health Outcomes (ECHO) research program. J. Pediatr. 196, 298-300 (2018).

8. Jacobson, L., Parker, C., Cella, D., Mroczek, D., \& Lester B., Program Collaborators for Environmental influences on Child Health Outcomes. SPR Perspectives: 
SPR Perspectives: scientific opportunities in the Environmental... ME Romano et al.

approaches to protocol standardization and data harmonization in the ECHOwide cohort study. Manuscript in preparation (2021).

9. ECHO: Environmental influences on Child Health Outcomes. Clinical Trials (ECHO IDeA States Pediatric Clinical Trials Network) https://echochildren.org/idea-statespediatric-clinical-trials-network/ (2021).

10. Hall, K. L. et al. The science of team science: a review of the empirical evidence and research gaps on collaboration in science. Am. Psychol. 73, 532-548 (2018).

11. Jacobson, L. P., Lau, B., Catellier, D. \& Parker, C. B. An Environmental Influences On Child Health Outcomes Viewpoint of data analysis centers for collaborative study designs. Curr. Opin. Pediatr. 30, 269-275 (2018).

12. Smith, B., Knox, S. \& Benjamin, D. K. Jr. Coordination of the Environmental influences on Child Health Outcomes program: so the whole is greater than the sum of its parts. Curr. Opin. Pediatr. 30, 263-268 (2018).

13. Blackwell, C. K. et al. Better sleep, better life? How sleep quality influences children's life satisfaction. Qual. Life. Res. https://doi.org/10.1007/s11136-020-02491-9 (2020).

14. McGeachie, M. J. Childhood asthma is a risk factor for the development of chronic obstructive pulmonary disease. Curr. Opin. Allergy Clin. Immunol. 17, 104-109 (2017).

15. Bui, D. S. et al. Childhood predictors of lung function trajectories and future COPD risk: a prospective cohort study from the first to the sixth decade of life. Lancet Respir. Med. 6, 535-544 (2018).

16. Costa, D. D., Pitrez, P. M., Barroso, N. F. \& Roncada, C. Asthma control in the quality of life levels of asthmatic patients' caregivers: a systematic review with metaanalysis and meta-regression. J. Pediatr. 95, 401-409 (2019).

17. Johnson, C. C. et al. Environmental influences on child health outcomes (ECHO) collaborators. US childhood asthma incidence rate patterns from the ECHO consortium to identify high-risk groups for primary prevention. JAMA Pediatr. e210667. https://doi.org/10.1001/jamapediatrics.2021.0667 (2021). Epub ahead of print.

18. Wang, A., Padula, A., Sirota, M. \& Woodruff, T. J. Environmental influences on reproductive health: the importance of chemical exposures. Fertil. Steril. 106, 905-929 (2016).
19. US Environmental Protection Agency. TSCA Chemical Substance Inventory https:// www.epa.gov/tsca-inventory (2020).

20. Buckley, J. P. et al. Program Collaborators for Environmental influences on Child Health Outcomes. Opportunities for evaluating chemical exposures and child health in the United States: the Environmental influences on Child Health Outcomes (ECHO) Program. J. Expo. Sci. Environ. Epidemiol. 30, 397-419 (2020).

21. Pellizzari, E. D. et al. Identifying and prioritizing chemicals with uncertain burden of exposure: opportunities for biomonitoring and health-related research. Environ. Health Perspect. 127, 126001 (2019).

22. Wright, R. O., Teitelbaum, S., Thompson, C. \& Balshaw, D. CHEAR Network. The Child Health Exposure Analysis Resource as a vehicle to measure environment in the Environmental influences on Child Health Outcomes Program. Curr. Opin. Pediatr. 30, 285-291 (2018).

23. Doherty, B. T., Pearce, J. L., Anderson, K. A., Karagas, M. R. \& Romano, M. E. Assessment of multipollutant exposures during pregnancy using silicone wristbands. Front. Public Health 8, 547239 (2020).

24. Lyall, K. et al. Distributional properties and criterion validity of a shortened version of the Social Responsiveness Scale: results from the ECHO program and implications for social communication research. J. Autism Dev. Disord. https://doi. org/10.1007/s10803-020-04667-1 (2020).

25. ECHO: Environmental influences on Child Health Outcomes. Mission and Guiding Principles https://echochildren.org/echos-guiding-principles/ (2021).

26. Conradt, E. et al. Prenatal opioid exposure: neurodevelopmental consequences and future research priorities. Pediatrics 144, e20190128 (2019).

27. ECHO: Environmental influences on Child Health Outcomes. ECHO Program's Response to COVID-19 https://echochildren.org/echo-programs-response-tocovid-19/ (2021).

28. National Institutes of Health, U.S. National Library of Medicine, Disaster Information Management Center. Disaster Lit. Environmental influences on Child Health Outcomes (ECHO) COVID-19 Questionnaires https://disasterinfo.nlm.nih.gov/ (2020). 